Received: 9 August 2018

Accepted: 9 January 2019

Published online: 27 February 2019
NTIFIC REP

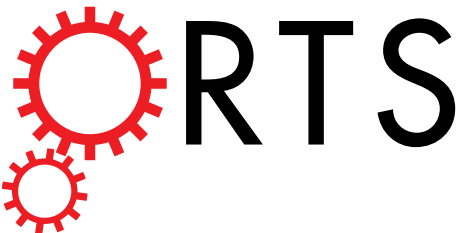

There are amendments to this paper

\title{
OPEN Inactivation of Ppp1r15a minimises weight gain and insulin resistance during caloric excess in female mice
}

Vruti Patel ${ }^{1,2}$, Guillaume Bidault ${ }^{3}$, Joseph E. Chambers ${ }^{1}$, Stefania Carobbio ${ }^{3}$, Angharad J. T. Everden ${ }^{1}$, Concepción Garcés ${ }^{1}$, Lucy E. Dalton ${ }^{1}$, Fiona M. Gribble ${ }^{3}$, Antonio Vidal-Puig ${ }^{3,4}$ \& Stefan J. Marciniak $\mathbb{D}^{1,2}$

Phosphorylation of the translation initiation factor elF2 $\alpha$ within the mediobasal hypothalamus is known to suppress food intake, but the role of the elF $2 \alpha$ phosphatases in regulating body weight is poorly understood. Mice deficient in active PPP1R15A, a stress-inducible elF2 $\alpha$ phosphatase, are healthy and more resistant to endoplasmic reticulum stress than wild type controls. We report that when female Ppp1r15a mutant mice are fed a high fat diet they gain less weight than wild type littermates owing to reduced food intake. This results in healthy leaner Ppp1r15a mutant animals with reduced hepatic steatosis and improved insulin sensitivity, albeit with a possible modest defect in insulin secretion. By contrast, no weight differences are observed between wild type and Ppp1r15a deficient mice fed a standard diet. We conclude that female mice lacking the C-terminal PP1-binding domain of PPP1R15A show reduced dietary intake and preserved glucose tolerance. Our data indicate that this results in reduced weight gain and protection from diet-induced obesity.

Mechanisms that govern energy balance have evolved to cope with periods of nutrient scarcity interspersed by times of plenty. The intake of excess calories and their storage in adipose tissue is one such mechanism, but it can become maladaptive in the face of a high calorie western diet. The resulting epidemic of obesity is impairing health in part through increasing the prevalence of type 2 diabetes. By targeting the pathways that promote a positive energy balance it may be possible to prevent obesity and reduce its complications.

The endoplasmic reticulum (ER) is an organelle responsible for the biosynthesis of lipids and the folding of secretory proteins ${ }^{1}$. If protein-folding homeostasis (so-called proteostasis) is threatened by the accumulation of misfolded proteins inside the ER, the cell experiences ER stress. Pathways that are triggered by ER stress are collectively called the unfolded protein response (UPR). Because ER stress is frequently observed in tissues that have accumulated excess lipids it is likely that the UPR is also involved in regulating positive energy balance and/ or its associated metabolic complications ${ }^{2}$.

The UPR comprises three parallel pathways activated by distinct ER stress sensors: IRE1, ATF6 and PERK 3 . PERK plays a critical role in maintaining the health of insulin-secreting beta-cells and so homozygous mutations of the PERK gene in Wolcott-Rallison syndrome manifest as early onset insulin-dependent diabetes ${ }^{4}$. In mice, inactivation of the Perk gene also causes early beta-cell death ${ }^{5}$. PERK is a member of a kinase family that selectively phosphorylates eIF $2 \alpha$ on serine 51 to P-eIF $2 \alpha$ to trigger the integrated stress response (ISR) ${ }^{6,7}$. This phosphorylation of eIF $2 \alpha$ inhibits protein synthesis by preventing translation initiation and thus reduces the load of new proteins entering the ER. When eIF $2 \alpha$ is phosphorylated, although the synthesis of most proteins is inhibited, the mRNA of the transcription factor ATF4 is preferentially translated ${ }^{8}$. This leads to the induction of PPP1R15A (also known as GADD34), which binds protein phosphatase 1 (PP1) and G-actin to dephosphorylate P-eIF $2 \alpha^{9,10}$. After a delay of several hours this restores normal levels of translation thus enabling the synthesis of targets of the UPR. However, in the context of chronic ER stress the recovery of protein synthesis mediated by PPP1R15A contributes to toxicity ${ }^{11,12}$.

${ }^{1}$ Cambridge Institute for Medical Research (CIMR), University of Cambridge Wellcome Trust/MRC Building, Hills Road, Cambridge, CB2 OXY, UK. ²Department of Medicine, University of Cambridge, Addenbrooke's Hospital, Hills Rd, Cambridge, CB2 0SP, UK. ${ }^{3}$ Metabolic Research Laboratories, Wellcome Trust-MRC Institute of Metabolic Science, Addenbrooke's Hospital, Cambridge, CB2 00Q, UK. "Wellcome Trust Sanger Institute, Wellcome Trust Genome Campus, Hinxton, CB10 1SA, UK. Correspondence and requests for materials should be addressed to S.J.M. (email: sjm20@cam.ac.uk) 
a

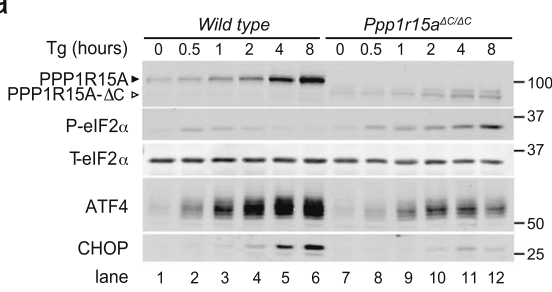

b

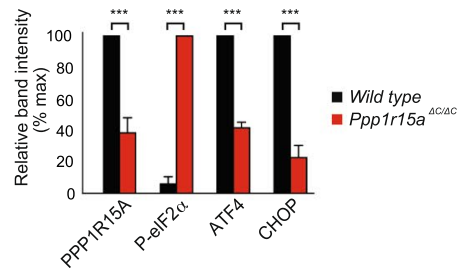

C

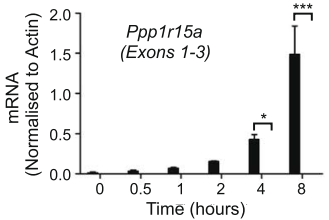

e

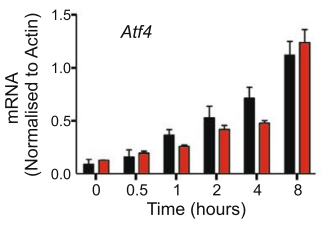

g

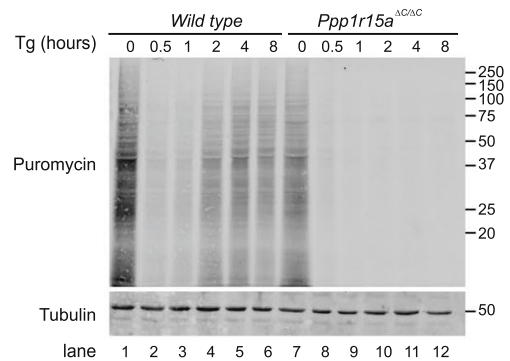

i

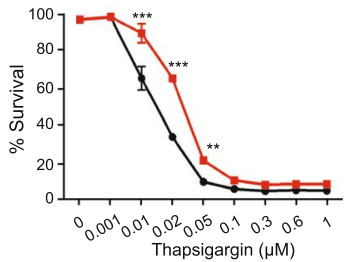

$\mathrm{k}$

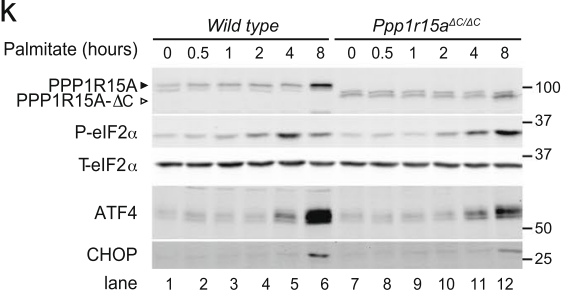

d
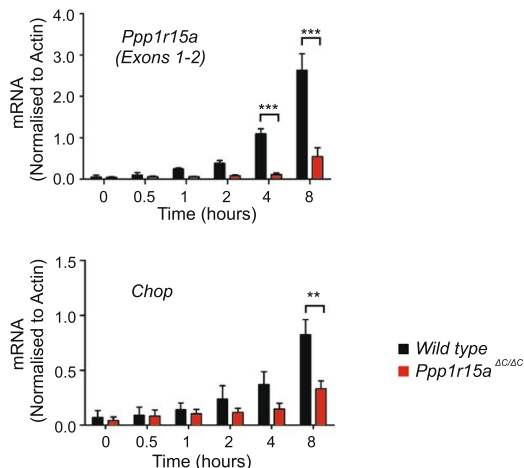

$\mathrm{h}$

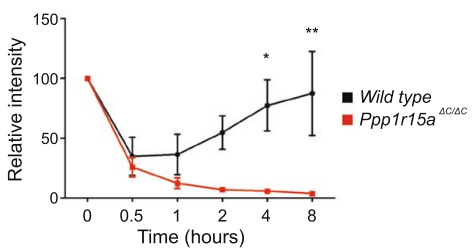

j

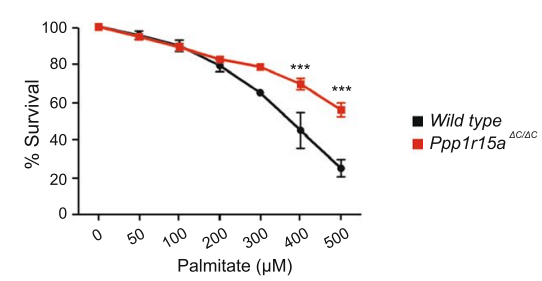

I

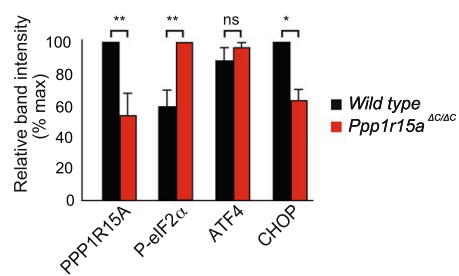

Figure 1. Inactivation of PPP1R15A reduces ER stress and protects against lipotoxicity in vitro. (a) Immunoblot for PPP1R15A, phosphorylated eIF2 $\alpha$ (P-eIF2 $\alpha$ ), total eIF2 $\alpha$ (T-eIF2 $\alpha$ ), ATF4 and CHOP in lysates of wild type and Ppp1r15a $a^{\Delta C / \Delta C}$ mouse embryonic fibroblasts (MEFs) following treatment with thapsigargin (Tg) $300 \mathrm{nM}$ for indicated times. Proteins of the expected sizes are marked with a solid triangle for PPP1R15A or an open triangle for PPP1R15A- $\triangle$ C. Molecular size markers shown in $\mathrm{kDa}$. (b) Quantification of (a) using ImageJ software. $\mathrm{N}=3$; mean \pm SEM; P value calculated by two-way ANOVA. (c-f) Wild type and Ppp1r15a ${ }^{\Delta C / \triangle C}$ MEFs were treated with thapsigargin $300 \mathrm{nM}$ for indicated times and RNA was prepared. Ppp1r15a (Exons 1-3), Ppp1r15a (Exons 1-2), Atf4, Chop mRNA were quantified relative to beta-actin by qRT-PCR. N=3; mean $\pm \mathrm{SEM}$; P value calculated by two-way ANOVA. (g) Immnoblot for puromycin (indicating rate of translation) and tubulin in lysates of wild type or Ppp1r15a $a^{\Delta C / \Delta C}$ MEFs following treatment with thapsigargin (Tg) $300 \mathrm{nM}$ for indicated times. Ten minutes prior to harvesting, puromycin was added to the culture medium at a final concentration of $10 \mathrm{ng} / \mathrm{mL}$. Molecular size markers shown in $\mathrm{kDa}$. (h) Immunoreactivity to puromycin within lysates served as a marker of protein translation and was quantified using ImageJ software. $\mathrm{N}=3$; mean $\pm \mathrm{SEM}$; $\mathrm{P}$ value calculated by two-way 
ANOVA with Bonferroni post hoc test. Molecular size markers shown in $\mathrm{kDa}$. (i) MTT assays were carried out to measure cell viability of wild type or Ppp $1 r 15 a^{\Delta C / \Delta C}$ MEFs following treatment with the indicated concentrations of thapsigargin for 48 hours. $\mathrm{N}=3$; mean $\pm \mathrm{SEM}$; P value calculated by two-way ANOVA with Bonferroni post hoc test. (j) MTT assays were carried out to measure cell viability of wild type or $P p p 1 r 15 a^{\Delta C / \Delta C}$ MEFs following treatment with the indicated concentrations of palmitate for 48 hours. $\mathrm{N}=3$; mean \pm SEM; $\mathrm{P}$ value calculated by two-way ANOVA with Bonferroni post hoc test. (k) Immunoblot for PPP1R15A, P-eIF2 $\alpha$, T-eIF2 $\alpha$, ATF4 and CHOP in lysates of wild type and Ppp $1 r 15 a^{\Delta C / \Delta C}$ mouse embryonic fibroblasts (MEFs) following treatment with palmitate $400 \mu \mathrm{m}$ for indicated times. Proteins of the expected sizes are marked with a solid triangle for PPP1R15A or an open triangle for PPP1R15A- $\triangle$ C. (1) Quantification of (A) using ImageJ software. N = 3; mean \pm SEM; P value calculated by two-way ANOVA. *** $\mathrm{p}<0.001, * * \mathrm{p}<0.01,{ }^{*} \mathrm{p}<0.05$.

We previously showed that inactivation of the Ppp1r15a gene generates phenotypically healthy, fertile mice that have increased resilience to ER stress ${ }^{12}$. Subsequently, loss of PPP1R15A has been shown to protect against tissue damage in a model of ER stress-induced disease ${ }^{13}$. Subtle reduction in the level of P-eIF2 $\alpha$, the substrate of PPP1R15A, in mice heterozygous for the $e I F 2 \alpha^{S 51 A}$ allele causes obesity, hyperleptinaemia and glucose intolerance when the animals are fed a high-fat diet ${ }^{14,15}$. However, while increased levels of eIF $2 \alpha$ phosphorylation within the hypothalamus have been shown to reduce food intake ${ }^{16}$, a recent report suggested that PPP1R15A deficient mice, which are impaired in P-eIF2 $\alpha$ dephosphorylation, spontaneously become obese ${ }^{17}$. The specific role of PPP1R15A in the regulation of energy balance therefore remains unclear.

We set out to determine the response of PPP1R15A deficient animals to caloric excess using a high-fat diet. Contrary to a previous report ${ }^{17}$, we observed $P p p 1 r 15 a^{\Delta C / \Delta C}$ mice to gain significantly less weight than wild type littermates when fed a high-fat diet. Metabolic phenotyping revealed reduced food intake in the absence of changes in energy expenditure. Consequently, despite having a likely subtle defect in insulin secretion, Ppp1r15a $a^{\Delta C / \Delta C}$ mice were resistant to diet induced obesity, remaining leaner and less insulin resistant than their wild type littermates fed on a high-fat diet.

\section{Results}

Inactivation of PPP1R15A reduces ISR signalling and protects from lipotoxicity in vitro. As expected, when mouse embryonic fibroblasts (MEFs) generated from wild type embryos were treated with the ER stressor thapsigargin, full length PPP1R15A protein was induced progressively over an 8-hour period (Fig. 1a,b). This mirrored the transcriptional induction of Ppp1r15a mRNA (Fig. 1c,d). In Ppp1r15a ${ }^{\Delta C / \Delta C}$ MEFs, PPP1R15A- $\triangle \mathrm{C}$ was induced over a similar time course, but to significantly lower levels. A primer pair targeting the deleted exon 3, served as a convenient control for genotype (Fig. 1c); while, primers within the remaining Ppp1r15a $a^{\Delta C}$ allele enabled us to use endogenous Ppp1r15a mRNA as a readout of the P-eIF2 $\alpha$-ATF4 (ISR) pathway. Ppp1r15a $a^{\Delta C / \Delta C}$ showed no induction of exon 3, but endogenous $P p p 1 r 15 a^{\Delta C}$ was induced albeit to a much lower level than the wild type allele (Fig. 1d). Consistent with this, P-eIF2 $\alpha$ peaked in wild type cells at 0.5 hours after treatment with thapsigargin, falling back to baseline by 8 hours as PPP1R15A levels rose, while P-eIF $2 \alpha$ rose monotonically in the Ppp1r15a $a^{\Delta C / \Delta C}$ MEFs (Fig. 1a,b). This confirmed that $P p p 1 r 15 a^{\Delta C / \Delta C}$ cells are impaired in the dephosphorylation of P-eIF2 $\alpha$ during ER stress.

The induction of ATF4 protein was significantly reduced in $P p p 1 r 15 a^{\Delta C / \Delta C}$ MEFs compared with wild type controls despite similar levels of Atf4 mRNA (Fig. 1a,b,e). This reflected a more intense inhibition of translation in Ppp1r15a ${ }^{\Delta C / \Delta C}$ MEFs (Fig. 1g,h). Consistent with this, cells treated with tunicamycin, which induces ER stress more slowly and so inhibits translation more weakly, showed no deficit of ATF4 in the Ppp1r15a $a^{\Delta C / \Delta C}$ MEFs (Supplementary Fig. 1). The lower levels of ATF4 in thapsigargin-treated $P p p 1 r 15 a^{\Delta C / \Delta C}$ cells reduced induction of its target gene Chop (Fig. 1f) and so contributed to the reduced induction of endogenous $P p p 1 r 15 a^{\Delta C}$ mRNA (Fig. 1d).

The reduction in ISR signalling in thapsigargin-treated $P p p 1 r 15 a^{\Delta C / \Delta C}$ cells correlated with reduced toxicity of this agent. When wild type and Ppp1r15a $a^{\Delta C / \Delta C}$ MEFs were treated for 48 hours with thapsigargin, PPP1R15A-deficient cells required significantly higher concentration of drug to reduce viability (Fig. 1i). The saturated free fatty acid palmitate causes cell death in part via the induction of ER stress ${ }^{18-21}$ and, like thapsigargin, this may involve the depletion of ER calcium ${ }^{18,22}$. We therefore tested whether inactivation of PPP1R15A would protect cells from the lipotoxicity of palmitate. Once again, the PPP1R15A-deficient cells required significantly higher levels of palmitate to reduce viability (Fig. 1j). Therefore, we concluded that deficiency of PPP1R15A reduced the lipotoxic insult induced by palmitate in vitro. As had been observed for thapsigargin, palmitate caused significantly higher levels of P-eIF2 $\alpha$ and lower levels of CHOP in Ppp1r15a ${ }^{\Delta C / \Delta C}$ MEFs than in controls (Fig. 1k,1).

Inactivation of PPP1R15A reduces weight gain of mice fed a high-fat diet and prevents diet-induced hepatic steatosis. Reduced phosphorylation of eIF2 $\alpha$ has been shown to enhance weight gain of mice fed a high-fat diet ${ }^{14}$. However, loss of the eIF2 $\alpha$ phosphatase PPP1R15A has also been found to cause obesity in male mice, but not in females ${ }^{17,23}$. To examine the effect of PPP1R15A expression in females in greater detail, we randomly assigned 6-week old female wild type and $P p p 1 r 15 a^{\Delta C / \Delta C}$ littermates to normal chow or $60 \%$ high-fat diet. Bodyweights were monitored weekly for the following 10 weeks (Fig. 2a). Strikingly, only when fed a $60 \%$ high-fat diet, Ppp1r15a $a^{\Delta C / \Delta C}$ animals gained less weight than their wild type littermates (Fig. 2a,b). This reduced weight did not arise from a runty phenotype, as body length was not significantly different between the two genotypes (Fig. 2c).

We then used time domain-NMR to investigate whether the body weight differences reflected differences in fat or lean mass. Lean mass was not different between the two genotypes at 6, 17 or 24 weeks (Fig. 2d). However, 
a
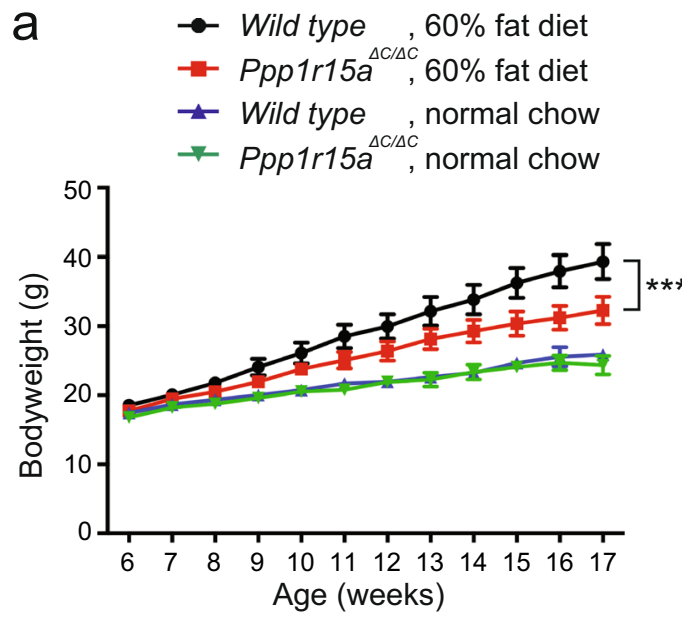

b

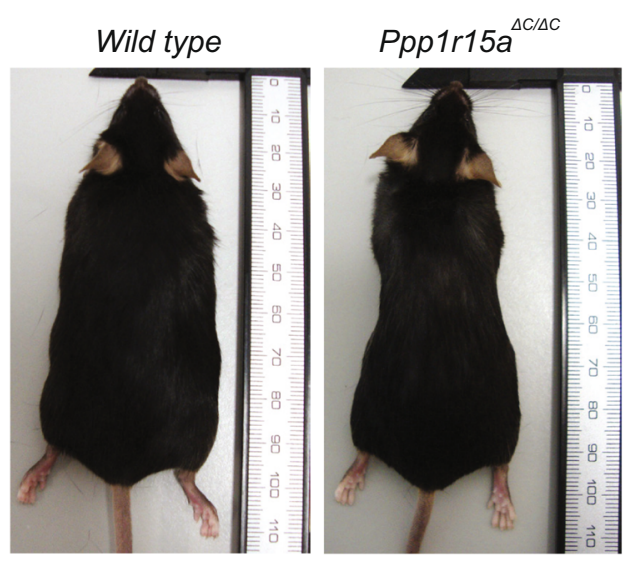

C

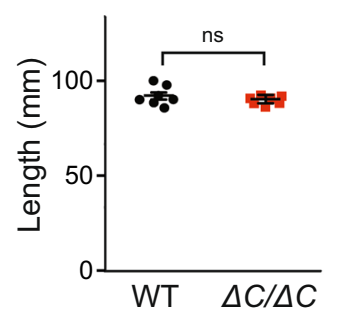

d

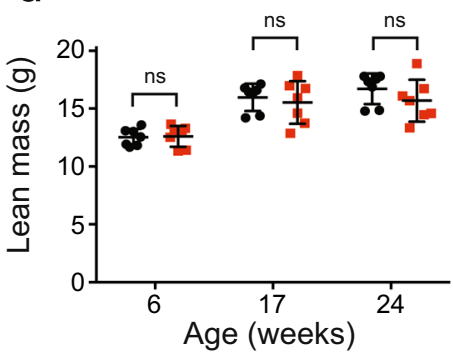

e

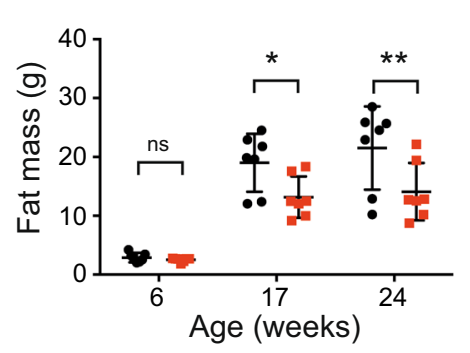

f
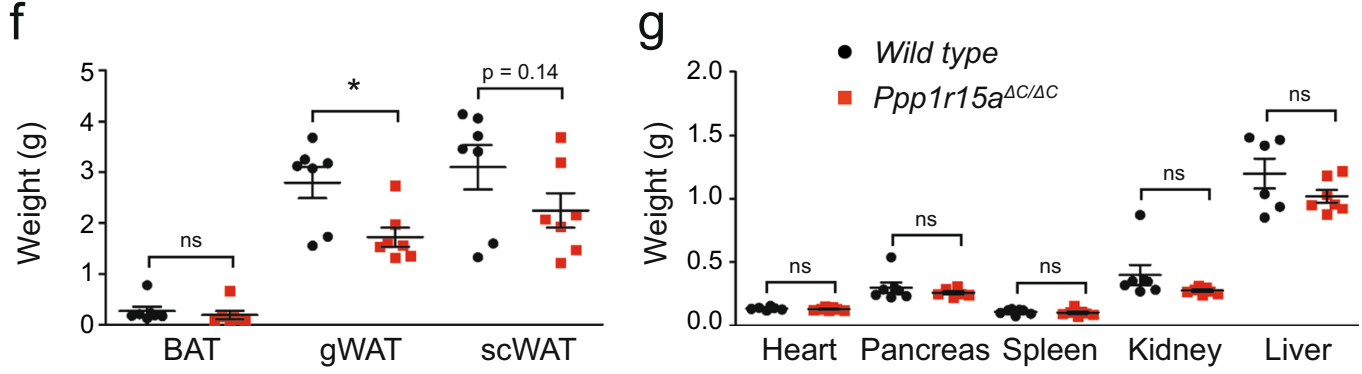

Figure 2. Inactivation of PPP1R15A reduces weight gain following a 60\% high-fat diet in vivo: (a) At 6 weeks of age, wild type and Ppp1r15a $a^{\Delta C / \Delta C}$ mice were fed either standard chow diet or $60 \%$ high-fat diet and their bodyweights measured. P value calculated by two-way ANOVA. (b) Representative images of wild type and Ppp1r15a $a^{\Delta C / \Delta C}$ mice fed a $60 \%$ high-fat diet for 18 weeks. (c) Length of each mouse was measured at time of harvest. (d,e) Using time domain nuclear magnetic resonance (TD-NMR), the (d) lean mass, and (e) fat mass of the mice were measured prior to high-fat feeding (6-weeks), during high-fat feeding (17-weeks) and at the end of the study (24-weeks). (f) The wet weights of each adipose tissue were measured at harvest. Brown adipose tissue (BAT); gonadal white adipose tissue (gWAT) and subcutaneous white adipose tissue (scWAT). (g) The organs of each mouse were also harvested at the end of the study and wet weights measured. $\mathrm{N}=7$; mean plotted \pm SEM; unpaired Student's unpaired t-test used for statistical analysis. ${ }^{* * *} \mathrm{p}<0.001, * * \mathrm{p}<0.01$, $* \mathrm{p}<0.05$.

although accretion of fat mass rose progressively in both groups, the increase was significantly attenuated in the Ppp1r15a $a^{\Delta C / \Delta C}$ animals at 17 and 24 weeks (Fig. 2e). Organ weight measurements at 24 weeks were not different between genotypes - only gonadal white adipose tissue mass was significantly reduced in the $P p p 1 r 15 a^{\Delta C / \Delta C}$ animals compared with the wild type group (Fig. 2f,g). The $1 \mathrm{~g}$ difference in the weight of subcutaneous white fat (Fig. 2f) could not account entirely for the difference in mean weight between the two groups ( $8 \mathrm{~g}$ at 17 weeks of age; Fig. 2a,b). Since the time domain-NMR showed this difference arose from fat rather than lean mass, it is likely that non-measured sources of fat, e.g. within the periteum, also contributed to the reduced corpulence of the Ppp1r15a ${ }^{\Delta C / \Delta C}$ animals compared with their sisters.

These findings confirmed that differences in bodyweight between the genotypes resulted from reduced fat expansion in the Ppp1r15a $a^{\Delta C / \Delta C}$ mice rather than differences in linear growth per se.

In accordance with a healthy lean phenotype observed in $\operatorname{Ppp} 1 r 15 a^{\Delta C / \Delta C}$ mice, histological analysis and neutral lipid staining revealed reduced hepatic steatosis in Ppp1r15a $a^{\Delta C / \Delta C}$ mice fed a high-fat diet compared to the wild type controls (Fig. 3a). It has previously been described that over-expression of PPP1R15A attenuates 
a

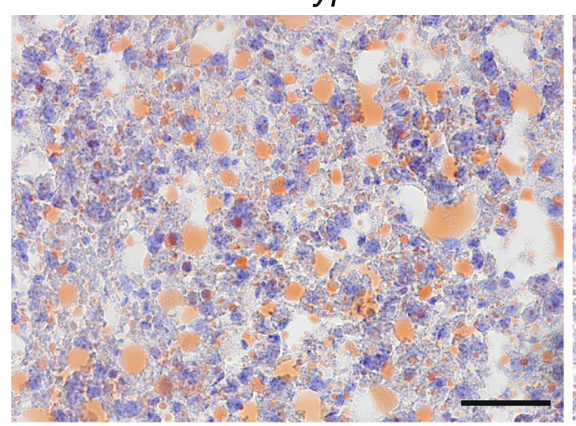

b

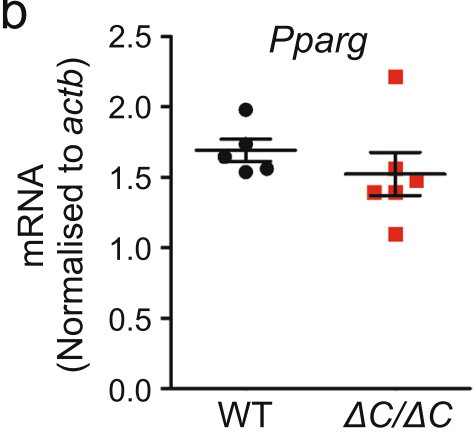

d

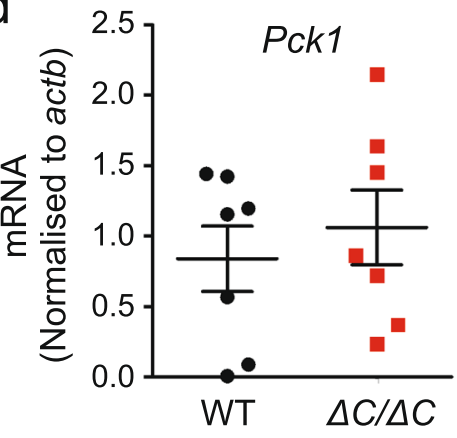

f

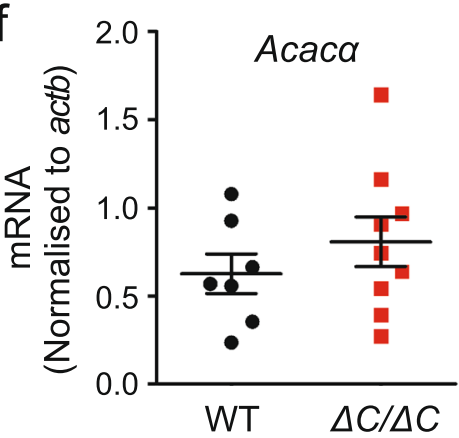

$\operatorname{Ppp} 1 \mathrm{r15a} \mathrm{a}^{\triangle \mathrm{C} / \Delta \mathrm{C}}$

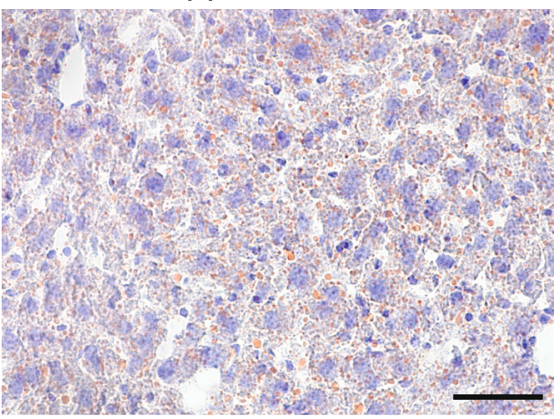

C

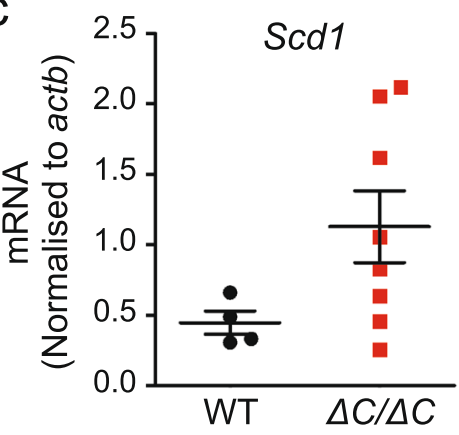

e

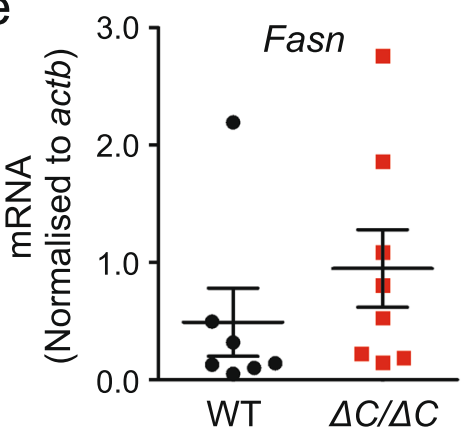

g

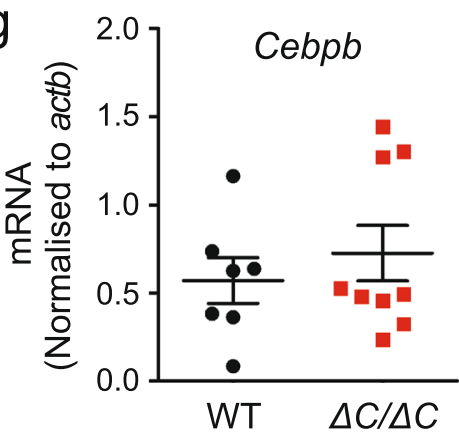

Figure 3. $P p p 1 r 15 a^{\Delta C / \Delta C}$ mice show reduced hepatic steatosis following high-fat feeding but no changes in expression of hepatic lipogenic enzymes. (a) To determine hepatic liver content, liver dissected from wild type and $P p p 1 r 15 a^{\Delta C / \Delta C}$ mice was frozen in liquid nitrogen. Fifteen-micrometre sections of liver were cut using a cryostat and stained using Oil Red O lipid stain and counterstained with haematoxylin (OROH). Representative images are shown. Scale bar: $50 \mu \mathrm{m}$. (b-g) RNA was prepared from liver taken from wild type (WT) and $P p p 1 r 15 a^{\Delta C / \Delta C}(\Delta \mathrm{C} / \Delta \mathrm{C})$ mice fed a high-fat diet for 12 weeks. Pparg, Scd1, Pck1, Fasn, Acaca, and Cebpb mRNA were quantified relative to actb by qRT-PCR. $\mathrm{N}=4-9$; mean plotted \pm SEM; none of the mRNA levels were significantly different by unpaired Student's unpaired t-test.

hepatic lipid biogenesis by preventing the accumulation of P-eIF $2 \alpha$ and thus skewing lipogenic transcriptional programmes $^{24}$. However, our analysis of the expression of genes involved in hepatic lipid metabolism in wild type and Ppp1r15a $a^{\Delta C / \Delta C}$ mice fed on high-fat diet for 12 weeks revealed no differences between the two genotypes in 
a

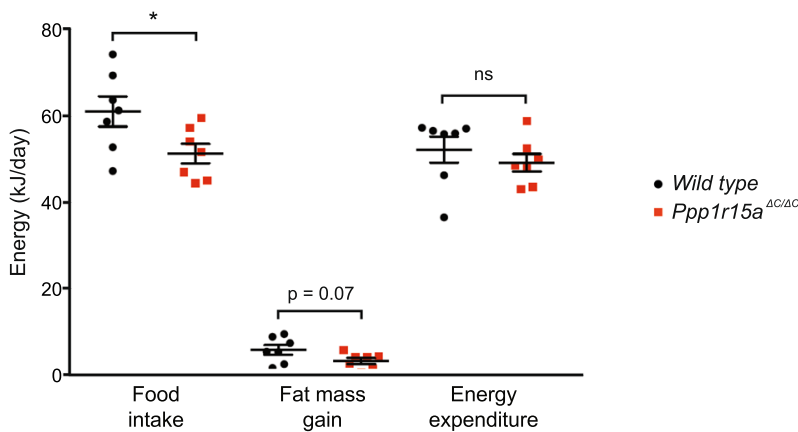

b
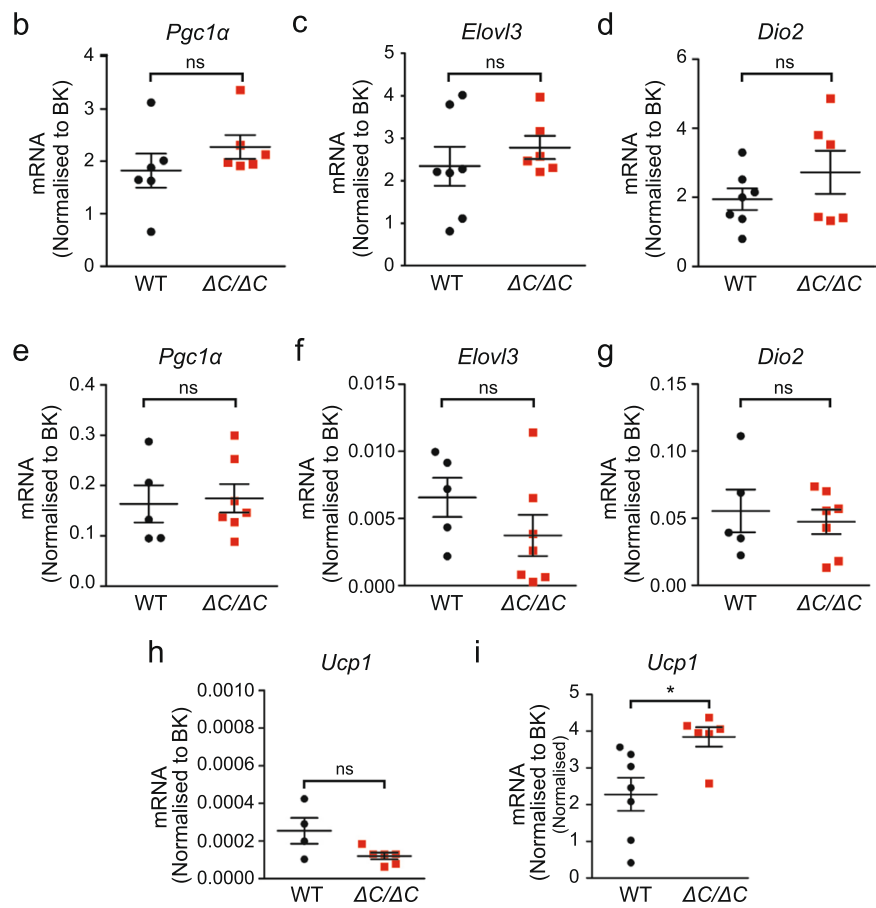

Elov/3

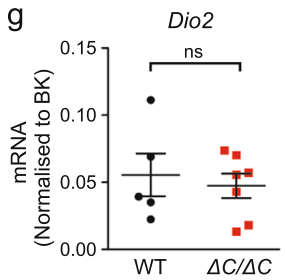

j
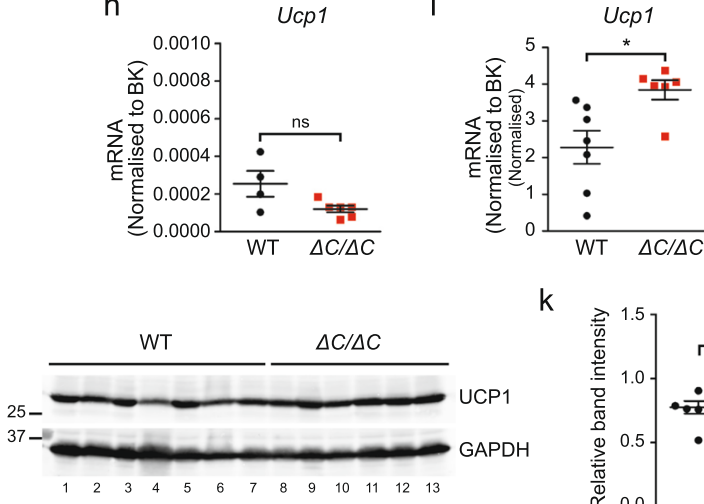

$\mathrm{k}$

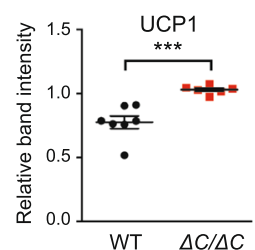

I
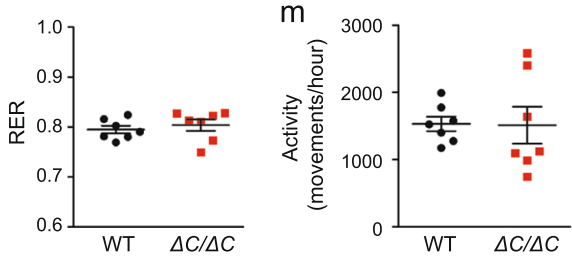

Figure 4. Metabolic phenotyping of wild type and $P p p 1 r 15 a^{\Delta C / \Delta C}$ mice indicate reduced food intake following high-fat feeding. (a) Mice were single housed at 17 -weeks old and food intake was measured three times a week, for 2-weeks. Following food intake measurements, indirect calorimetry was carried out over 48 hours. Food intake, fat mass gain and energy expenditure were calculated. RNA was prepared from brown adipose tissue taken from wild type (WT) and $\operatorname{Ppp} 1 r 15 a^{\Delta C / \Delta C}(\Delta \mathrm{C} / \Delta \mathrm{C})$ mice fed a high-fat diet for 18 weeks. $\mathrm{P}$ value calculated by unpaired Student's t-test. (b-d) Pgc1a, Elovl3 and Dio2 mRNA were quantified relative to the geometrical mean of $a c t b, 36 b 4,18 S$ and $B 2 M$ by qRT-PCR referred to as BestKeeper (BK). RNA was prepared from subcutaneous brown adipose tissue taken from wild type and $P p p 1 r 15 a^{\Delta C / \Delta C}$ mice fed a high-fat diet for 18 weeks. (e-g) Pgc1a, Elovl3 and Dio2 mRNA were quantified relative to the geometrical mean of actb, 36b4, 18S and $B 2 M$ by qRT-PCR referred to as BK analysis. RNA was prepared from white adipose tissue taken from wild type and $P p p 1 r 15 a^{\Delta C / \Delta C}$ mice fed a high-fat diet for 18 weeks. (h) RNA was prepared from subcutaneous white adipose tissue taken from wild type and $P p p 1 r 15 a^{\Delta C / \Delta C}$ mice fed a high-fat diet for 18 weeks and Ucp1 mRNA was quantified relative to $a c t b, 36 b 4,18 S$ and $B 2 M$ by qRT-PCR referred to as BK. (i) RNA was prepared from 
brown adipose tissue taken from wild type and $P p p 1 r 15 a^{\Delta C / \Delta C}$ mice fed a high-fat diet for 18 weeks and $U c p 1$ mRNA expression was quantified relative to $a c t b, 3664,18 S$ and $B 2 M$ by qRT-PCR and BK analysis. (j) Immunoblot for UCP1 and GAPDH of tissue lysates prepared from brown adipose tissue harvested from wild type and Ppp1r15a $a^{\Delta C / \Delta C}$ mice fed a high-fat diet for 18 weeks. Molecular size markers shown in $\mathrm{kDa}$. (k) Quantification of (D) using ImageJ software. $\mathrm{N}=4-7$, mean band intensity plotted \pm SEM; unpaired Student's t-test used for statistical analysis. $(\mathbf{l}, \mathbf{m})$ Indirect calorimetry was also used to measure the (l) respiratory exchange ratio and $(\mathbf{m})$ activity of the mice over 48 hours. $\mathrm{N}=7$; mean plotted $\pm \mathrm{SEM}$; unpaired Student's t-test used for statistical analysis. $* * * \mathrm{p}<0.001,{ }^{*} \mathrm{p}<0.05$.

Pparg, Scd1, Pck1, Fasn and Acaca (Fig. 3b-f). Furthermore, expression of Cebpb, a positive regulator of hepatic Ppary ${ }^{25,26}$, was similar in both genotypes (Fig. 3g). These results suggest that the reduced hepatic steatosis of Ppp1r15a $a^{\Delta C / \Delta C}$ mice was related to a healthy phenotype resulting from decreased exogenous input and reduced adiposity.

Ppp1r15a mutant animals consume less high-fat food without altering energy expenditure. We then investigated the factors contributing to the resistance of $P p p 1 r 15 a^{\Delta C / \Delta C}$ mice to high-fat diet-induced obesity. As shown in Fig. 2, by 12 weeks on a high-fat diet the bodyweights of wild type and $\operatorname{Ppp} 1 r 15 a^{\Delta C / \Delta C}$ mice were diverging. Therefore at 12 weeks, food intake of singly-housed mice was measured over a 2-week period. This was followed by metabolic rate measurements using indirect calorimetry for a further 48 hours. Food intake, fat mass gained and energy expenditure were calculated as total energy per day for each mouse (Fig. 4a). Ppp1r15a $a^{\Delta C / \Delta C}$ mice showed a significant $16 \%$ reduction in food intake compared with wild type controls indicating that inactivation of PPP1R15A selectively decreased the consumption of the high-fat diet (Fig. $4 \mathrm{a}$ ). In the absence of a conditional allele of Ppp1r15a it remains to be determined if these differences stem from a function of PPP1R15A in central or peripheral tissues. With respect to energy dissipation, we observed no significant differences in energy expenditure between the two genotypes, nor differences in the expression of the thermoregulatory genes, $P g c 1 \alpha$, Elovl3 and Dio2, either in brown (Fig. 4b-d) or white adipose tissue (Fig. 4e-g). In the brown adipose tissue, we observed a small increase in Ucp1 mRNA and protein (Fig. 4h-k), which was not associated with a decreased respiratory exchange ratio (RER) or an increase in energy expenditure, indicating no preferential oxidation of fatty acids in the Ppp $1 r 15 a^{\Delta C / \Delta C}$ mice (Fig. 4l). These results argue against PPP1R15A having a major impact on the thermogenic activity of brown and subcutaneous white adipose tissue. Additionally, physical activity rates were indistinguishable between the genotypes (Fig. $4 \mathrm{~m}$ ). Thus, the main contributor to resistance to weight gain was decreased food consumption.

Glucose tolerance and insulin tolerance testing in wild type and $P p p 1 r 15 a^{\Delta C / \Delta C}$ mice. Reduced body weight is not always a sign of health as it is observed in animals with primary adipose tissue dysfunction or lipodystrophy. Thus it was important to determine if the differences in weight gain were associated with improved glucose homeostasis in $P p p 1 r 15 a^{\Delta C / \Delta C}$ mice. The glucose tolerance test of mice fed a high-fat diet unexpectedly revealed a hyperglycaemic response in $P p p 1 r 15 a^{\Delta C / \Delta C}$ animals at 20 and 30 minutes following the intraperitoneal injection of glucose (Fig. 5a). This suggested that despite being leaner, the $P p p 1 r 15 a^{\Delta C / \Delta C}$ mice were slightly less glucose tolerant than their wild type littermates. This counterintuitive effect was relatively modest, as the areas under the blood glucose curves were not significantly different between wild type and $P p p 1 r 15 a^{\Delta C / \Delta C}$ mice (Fig. 5b). Thus, this could have been the result of insulin resistance or impaired insulin secretion by the Ppp1r15a $a^{\Delta \mathrm{C} / \Delta \mathrm{C}}$ mice, although basal and 30 minute insulin levels after glucose administration were not measurably different (Fig. 5c).

To determine their insulin sensitivity, we performed an insulin tolerance test on animals fed a high-fat diet. Following the injection of $0.75 \mathrm{U} / \mathrm{kg}$ of insulin, blood glucose fell in both groups, although to a significantly greater degree in the Ppp1r15a $a^{\Delta C / \Delta C}$ mice (Fig. 5e,f). This was seen as both a lower nadir in glucose level and a significantly different area under the blood glucose curve. In line with their leaner phenotype, basal levels of circulating free fatty acids in high-fat diet-fed $P p p 1 r 15 a^{\Delta C / \Delta C}$ mice were also significantly lower compared to wild type controls (Fig. 5d). This indicated that, in keeping with their leanness and reduced free fatty acid levels, Ppp1r15a $a^{\Delta C / \Delta C}$ animals were more insulin sensitive than their wild type controls. This further suggested a defect in insulin secretion since Ppp1r15a $a^{\Delta C / \Delta C}$ mice were less glucose tolerant than their wild type littermates.

Of note, although the basal levels of fasting circulating insulin were not significantly different between the two genotypes (Fig. 5c), western blot analysis revealed higher levels of insulin within the pancreata of unfasted Ppp1r15a $a^{\Delta C / \triangle C}$ animals compared with wild type controls (Fig. $5 \mathrm{~g}, \mathrm{~h}$ ) despite similar levels of insulin mRNA (Fig. 5i,j). This also suggested a defect in the secretion of insulin. Loss of PPP1R15A therefore caused opposing effects on glucose homeostasis: on the one hand improving insulin sensitivity, most likely via a negative energy balance, while also impairing early phase insulin secretion allowing higher peak glucose levels following glucose challenge.

Inactivation of PPP1R15A improves glucose tolerance in a model of beta-cell exhaustion. To determine if inactivation of PPP1R15A would have a net beneficial or detrimental effect in metabolic disease, we set out to model the later stages of type 2 diabetes wherein beta-cell mass falls in the face of sustained insulin resistance ${ }^{27}$. To achieve this, we fed mice a high-fat diet then treated with the beta-cell toxin streptozotocin at a dose optimized to cause subtotal beta-cell loss. At age 6 weeks, mice were placed on $60 \%$ high-fat diet and at 12 weeks were injected with $50 \mathrm{mg} / \mathrm{kg}$ streptozotocin on five consecutive days to mimic the loss of beta-cell mass seen in advanced type 2 diabetes ${ }^{27}$. As expected, streptozotocin injection induced weight loss (Fig. 6a) and a 
a

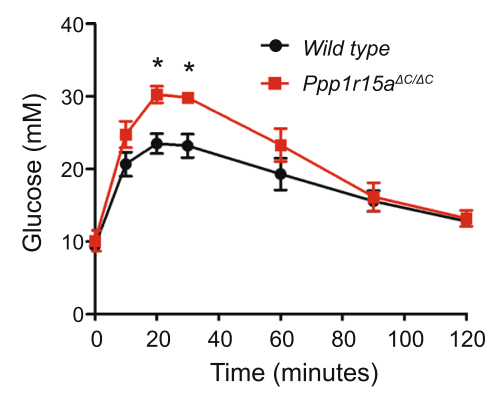

C

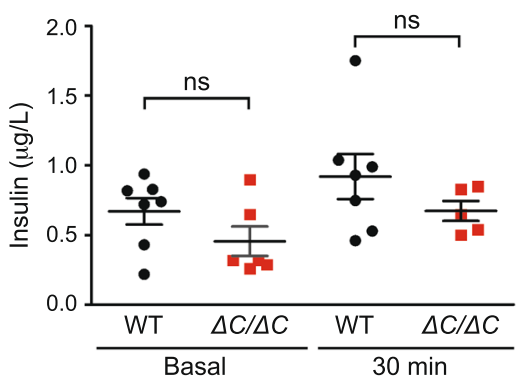

e

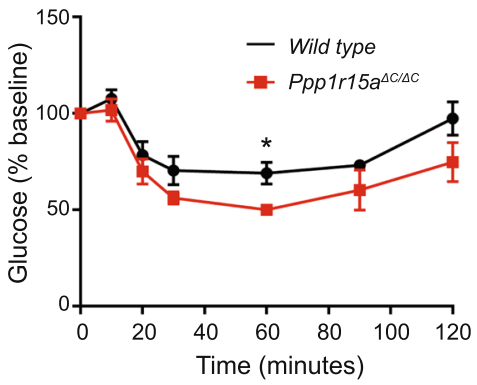

g

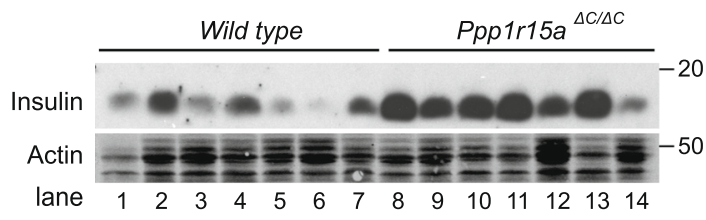

i

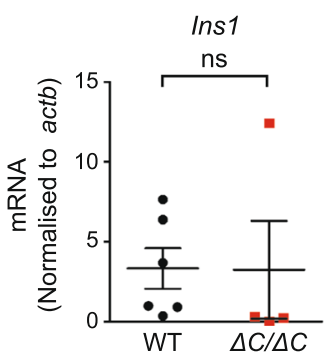

b

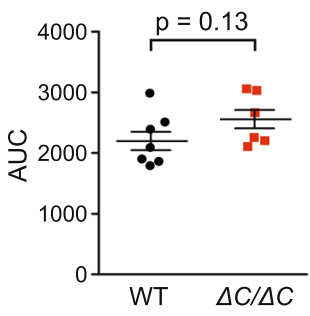

d

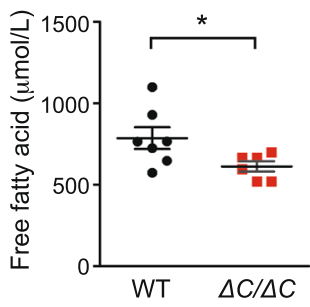

f

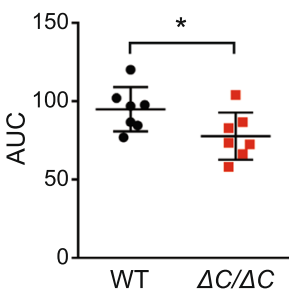

$\mathrm{h}$
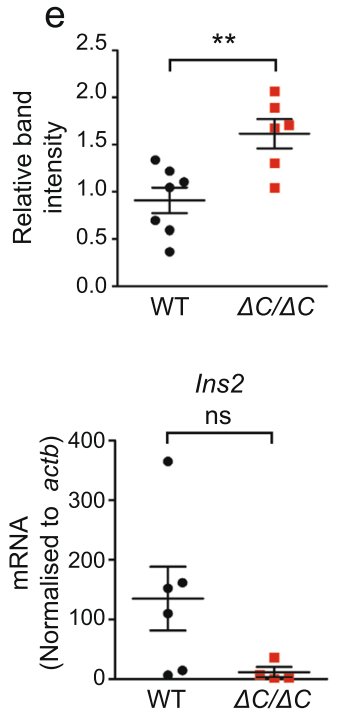

Figure 5. Glucose tolerance and insulin tolerance testing of wild type and $P p p 1 r 15 a^{\Delta C / \Delta C}$ mice. (a) At age 22 weeks (16 weeks on high-fat diet), the mice were fasted for 16 hours, after which mice were intraperitoneally injected with $1 \mathrm{~g} / \mathrm{kg}$ glucose, where the dose used was calculated using the average bodyweight of the full cohort of mice. Glucose levels were then measured at 10, 20, 60, 90 and 120 minutes post- injection. P value was calculated by two-way ANOVA with post hoc Bonferroni test. (b) Quantification of the glucose tolerance test (GTT) carried out using the area under the curve (AUC). (c) Blood samples were collected at 0 minutes (basal), prior to glucose injection and at 30 minutes post-injection. Serum was separated out and measured for insulin. P value calculated by unpaired Student's t-test. (d) Free fatty acid levels in the basal serum sample were also measured. P value calculated by unpaired Student's t-test. (e) At age 23 weeks (17 weeks on high-fat diet), the mice were fasted for 4 hours, after which mice were intraperitoneally injected with $0.75 \mathrm{U} / \mathrm{kg}$ insulin, where the dose used was calculated using the average bodyweight of the full cohort of mice. Glucose levels were then measured at 10,20,60, 90 and 120 minutes post-injection. P value was calculated by two-way ANOVA with post hoc Bonferroni test. (f) Quantification of the insulin tolerance test (ITT) was carried out using the AUC. P value 
calculated by unpaired Student's t-test. (g) Immunoblot for insulin and actin in protein lysates prepared from whole pancreas extracted from wild type and $P p p 1 r 15 a^{\Delta C / \Delta C}$ mice fed a high-fat diet for 18 weeks.

(h) Quantification of (g) using ImageJ software. P value calculated by unpaired Student's t-test. Molecular size markers shown in $\mathrm{kDa}$. (i-j) Total RNA was extracted from whole pancreas of wild type and $P p p 1 r 15 a^{\Delta C / \Delta C}$ mice fed a high-fat diet for 18 weeks. Ins 1 and Ins 2 mRNA were quantified relative to $a c t b$ by qRT-PCR. $\mathrm{N}=4-7$; mean plotted \pm SEM; unpaired Student's unpaired t-test used for statistical analysis.

progressive increase in fasting glucose in both groups (Fig. 6b). Immunofluorescence staining confirmed that islet morphology appeared similar between the genotypes prior to injection with streptozotocin, and both genotypes responded with similar reductions in islet size and falls in circulating insulin (Fig. 6c,d). However, when challenged with an intraperitoneal injection of glucose, the $P p p 1 r 15 a^{\Delta C / \Delta C}$ mice were significantly more glucose tolerant than wild type animals (Fig. 6e). As expected, both genotypes had higher peak glucose levels compared with animals not treated with streptozotocin (compare Figs $5 \mathrm{a}$ and $6 \mathrm{e}$ ), but despite having a partial depleted beta-cell mass, $P p p 1 r 15 a^{\Delta C / \Delta C}$ mice no longer showed higher peak glucose levels compared to wild type controls, but still displayed an increased glucose clearance (Fig. 6e).

\section{Discussion}

We have shown that female mice lacking functional PPP1R15A eat less and gain less weight than wild type animals when fed a high-fat diet. This leads to reduced fat mass, improved insulin sensitivity, lower levels of circulating free fatty acids, and less hepatic steatosis in the $P p p 1 r 15 a^{\Delta C / \Delta C}$ female mice.

Our observation that female Ppp1r15a $a^{\Delta C / \Delta C}$ mice remained leaner than controls on a high-fat diet was unexpected. The lean phenotype of these animals arises mainly from reduced food intake rather than increased energy expenditure, despite the observation that UCP1 expression is mildly increased in the brown adipose tissue (BAT) of Ppp1r15a $a^{\Delta C / \Delta C}$ mice. Importantly, the changes in UCP1 mRNA and protein levels do not necessarily result in changes in energy expenditure. For example, the Ucp1 knockout mouse has normal energy expenditure when reared at room temperature ${ }^{28}$. This occurs because energy expenditure in mice is a function of heat dissipation to the environment and the requirement to replace lost heat to maintain euthermia; this heat does not have to be derived from BAT. Given the normal energy expenditure in our model, the alterations in UCP1 may indicate a BAT-specific effect of P-eIF $2 \alpha$ on the regulation of UCP1 expression and therefore protein levels that deserves further investigation in the future.

Despite reporting this allele more than a decade ago, we had never observed a weight phenotype in this line ${ }^{12}$. However, we had not previously challenged these animals with a high-fat diet. Likewise, the recent report that deletion of the Ppp1r15a gene generates male mice that spontaneously become obese and develop fatty liver was surprising to us $^{17}$. In that study, Ppp1r15a deficient mice fed on standard chow (CLEA Rodent Diet CE-2) gained more weight than wild type controls, but this became significant only from 6 months of age - much older than is typical for our mice to be kept ${ }^{17}$. By contrast, a subsequent report by the same group failed to show excess obesity in female Ppp1r15a deficient mice even by 11-20 months of age ${ }^{23}$. Indeed, at 11-20 months of age on standard chow, 12 of 22 Ppp1r15a deficient females were described as being "lean", compared with 0 of 37 wild type animals (Table 1 in reference ${ }^{23}$ ). This led us to wonder if female Ppp1r15a deficient mice might display a more subtle weight phenotype than had been reported for males. We therefore examined the effects of normal chow versus high-fat diets, which revealed reduced weight gain in Ppp1r15a deficient females only when fed a high-fat diet.

A possible explanation for the dichotomous effects of Ppp1r15a loss in female vs male mice might involve the effects of oestradiol on hypothalamic lipotoxic ER stress. Reduced oestradiol levels following surgical ovariectomy lead to weight gain through hyperphagia and reduced energy expenditure in female mice ${ }^{29}$. Restoration of body weight following oestradiol replacement has recently been shown to involve a reduction of ER stress-causing ceramides within the ventromedial hypothalamus ${ }^{30}$. Similarly, inhibition of ceramide synthesis reduces hypothalamic ER stress and ameliorates weight gain following ovariectomy. It is tempting therefore to speculate that female mice are more susceptible to the weight reducing effects of Ppp1r15a loss because both oestradiol and P-eIF2 $\alpha$ are predicted to attenuate ER stress within the hypothalamus. A limitation of our present study is the lack of head to head comparison of male and female mice. Future studies will be necessary to address any potential interaction between PPP1R15A and oestradiol.

Differences between our results and those of Nishio and colleagues ${ }^{17,23}$ might also relate to the different alleles used. PPP1R15A is a large protein of $>600$ amino acids but only its C-terminal 150 residues are required for its phosphatase activity. We recently described how this C-terminal domain is both necessary and sufficient to interact with PP1 and actin to form a functional eIF $2 \alpha$ holophosphatase ${ }^{10,31}$. The different weight phenotypes between our Ppp1r15 $a^{\Delta C / \Delta C}$ mouse versus the complete-deletion mouse may hint to the existence of, as yet, unidentified functions of the N-terminal portion of PPP1R15A. To test this, future strain-controlled studies will need to compare these two alleles in head-to-head experiments.

In keeping with the healthy phenotype of our Ppp1r15a ${ }^{\Delta C / \Delta C}$ mice, no pathogenic mutations in $P P P 1 R 15 A$ have yet been reported in humans. Importantly, mutation of $P P P 1 R 15 A$ has not been identified in obese or lipodystrophic patients. By contrast, loss-of-function mutations of the constitutively expressed paralogue PPP1R15B have been shown to cause developmental defects both in mice ${ }^{32}$ and humans ${ }^{33}$. Mice homozygous for an inactive mutant allele of Ppp1r15b survive gestation but then die during the neonatal period ${ }^{32}$. Recently, two siblings with early onset diabetes, short stature, microcephaly and learning disability were reported to have a homozygous mutation of the PPP1R15B gene ${ }^{33}$. The mutation affected a conserved arginine in the C-terminal PP1-binding domain of PPP1R15B and impaired formation of an active eIF2 $\alpha$ holophosphatase when expressed in vitro. Although the mechanism by which PPP1R15B inactivation led to beta-cell dysfunction is unclear, it predicts that 
a

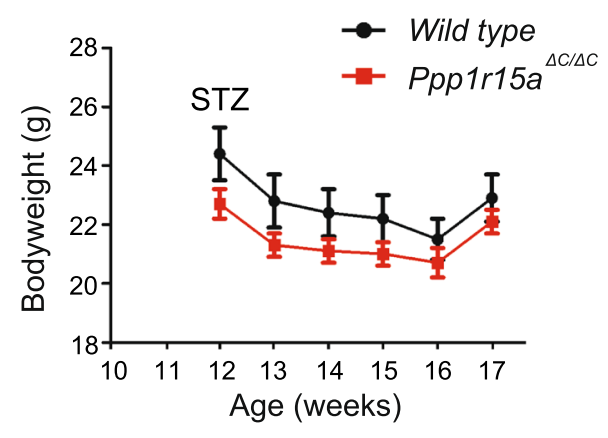

C

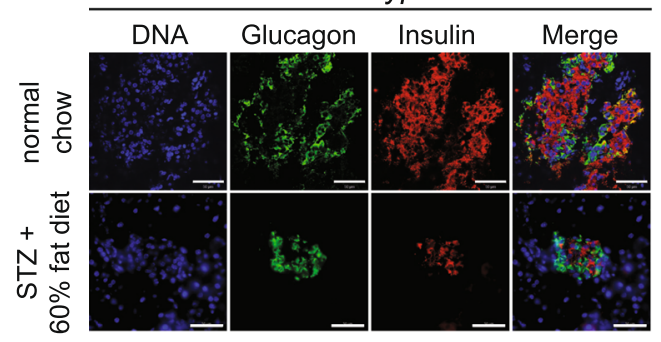

b

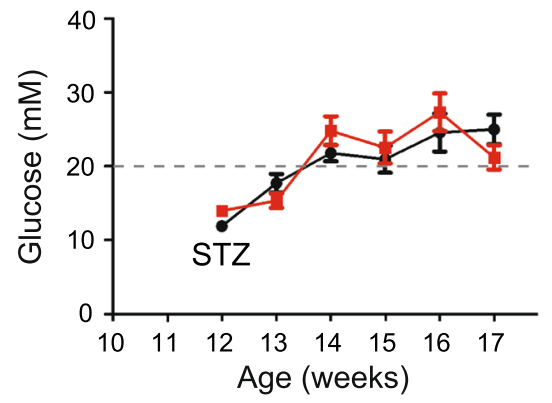

Ppp1r15a

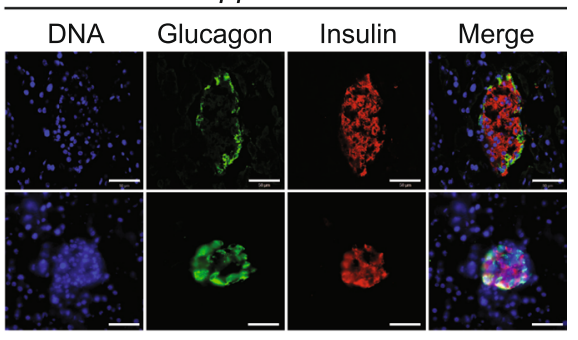

d
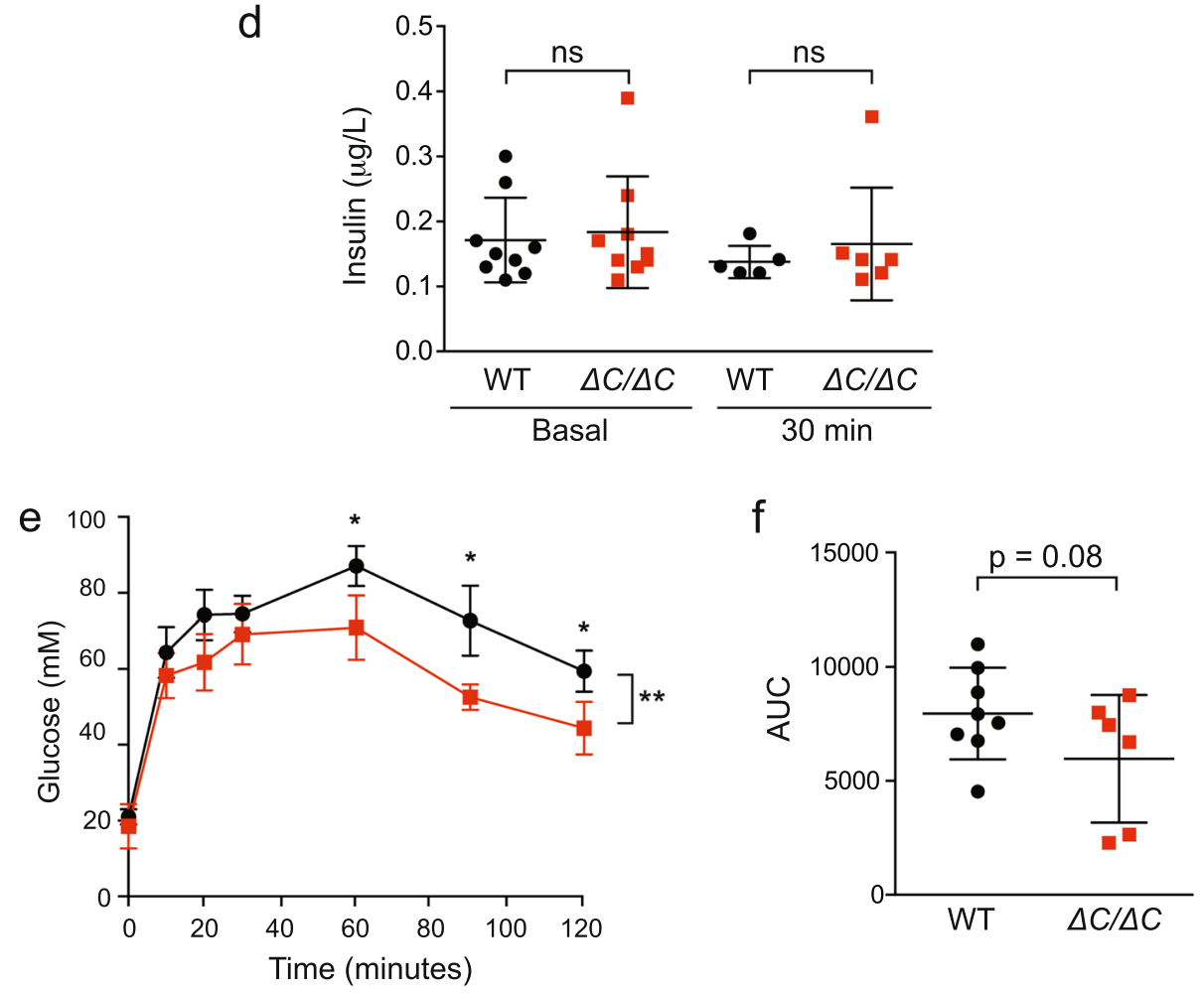

Figure 6. Induction of diabetes and measurement of pancreas function following high-fat feeding and streptozotocin treatment in wild type and Ppp1r15a $a^{\Delta C / \Delta C}$ mice. (a) Six-week old mice were put on a $60 \%$ high-fat diet for six weeks. At age 12 weeks, mice were injected with $50 \mathrm{mg} / \mathrm{kg}$ streptozotocin (STZ) on 5 consecutive days. Bodyweights of the mice were recorded for 5 weeks post STZ treatment. (b) Non-fasted blood glucose of the mice was measured for 5 weeks post STZ treatment. Clinical signs of diabetes assessed as mice with blood glucose levels above $20 \mathrm{mM}$ (marked with grey dashed line). (c) Fluorescence microscopy images of pancreas sections taken from wild type and $\operatorname{Ppp} 1 \mathrm{r} 15 a^{\Delta C / \Delta C}$ mice fed either a normal chow diet (top panel) or $60 \%$ high-fat diet and streptozotocin treated (bottom panel). Fixed tissue sections were probed with antibodies against glucagon (green) and insulin (red), and counterstained with Hoescht (blue) and imaged by confocal microscopy. Scale bar $=50 \mu \mathrm{m}$. (d) Circulating insulin levels of STZ-treated mice fed on a high-fat diet and shown compared to results from Fig. 5c. (e) Glucose tolerance test (GTT) was carried out on 17-week, $\mathrm{HFD}+\mathrm{STZ}$ treated mice. Mice were fasted overnight, and then injected with $1 \mathrm{~g} / \mathrm{kg}$ glucose. Blood glucose was 
measured at 0,10, 20, 30, 60, 90 and 120 minutes. N=7-9; two-way ANOVA and Bonferroni post-hoc test used for statistical analysis. (f) Quantification of the GTT carried out using the area under the curve (AUC). P value calculated by unpaired Student's t-test. $* \mathrm{p}<0.05$.

non-selective inhibition of P-eIF2 $\alpha$ dephosphoryaltion might have negative developmental effects. This highlights the need to develop selective PPP1R15A inhibitors.

ER stress links obesity, lipotoxicity and the development of insulin resistance ${ }^{34,35}$. Professional secretory cells, such as insulin secreting beta-cell, are prone to ER stress because they face large swings in the flux of new proteins traversing through their $\mathrm{ER}^{36}$. This in turn renders beta-cells especially vulnerable to defects in the UPR signaling pathway ${ }^{5}$. Indeed, mutations that impair the ability of mice or humans to phosphorylate eIF2 $\alpha$ during ER stress have disproportionately greater effects on the beta-cell compared to other secretory tissues ${ }^{4,5,14,37}$. Because many solid tumours also depend upon eIF2 $\alpha$ phosphorylation by PERK to withstand their hypoxic microenvironment $^{38}$, PERK inhibition has emerged as a potential chemotherapeutic strategy, but in pre-clinical models PERK inhibitors rapidly induce beta-cell toxicity, underling the reliance of this cell type on its ability to phosphorylate eIF $2 \alpha^{39,40}$. The precise mechanisms linking perturbed eIF2 $\alpha$ phosphorylation with beta-cell toxicity are not fully understood, but recent observations suggest that proper levels of P-eIF2 $\alpha$ are required to maintain appropriate levels of the chaperones required for proinsulin proteostasis ${ }^{41}$.

Phosphorylation of eIF2 $\alpha$ has complex effects on energy metabolism. When transgenic mice were generated to overexpress the C-terminal PP1 binding domain of PPP1R15A in the liver, they remained leaner than controls when fed a high-fat diet, and showed enhanced glucose tolerance, improved insulin sensitivity and reduced hepatic steatosis ${ }^{24}$. It is noteworthy, however, that phosphorylation of eIF $2 \alpha$ had a strikingly biphasic effect on hepatic lipid metabolism with lipogenic gene expression being suppressed both at reduced and at elevated levels of P-eIF $2 \alpha^{24}$. In addition, loss of hepatic P-eIF $2 \alpha$ by overexpression of PPP1R15A has been shown to affect muscle and adipose insulin sensitivity, possibly through altered levels of circulating IGFBP- $3^{42}$. Our observations now indicate that P-eIF2 $\alpha$ may also affect energy metabolism through effects on dietary intake.

Our observation that $P p p 1 r 15 a^{\Delta C / \Delta C}$ mice fed a high-fat diet consume significantly fewer calories compared with controls is consistent with a central effect of elevating P-eIF2 $\alpha$. This is in keeping with the known role of eIF $2 \alpha$ in regulating feeding behaviors. Aversion to diets imbalanced in amino acids has been shown to involve GCN2-mediated phosphorylation of eIF2 $\alpha$ in the brain, while reduced eIF2 $\alpha$ phosphorylation appeared to correlate with increased food intake ${ }^{43}$. Moreover, pharmacological enhancement of P-eIF2 $\alpha$ in the mediobasal hypothalamus with salubrinal has been shown to reduce food intake ${ }^{16}$. Central ER stress has also been implicated in mediating hepatic effects in diet-induced obesity in mice, since overexpression of the chaperone Grp78 in the circumventricular subfornical organ reduced hepatic steatosis without affecting weight gain, food intake or adiposity $^{44}$. However, we are unable to exclude an effect of $P p p 1 r 15 a^{\Delta C / \Delta C}$ on the secretion of orexigenic or anorexigenic hormones that might regulate dietary choice.

Owing to the absence of a conditional knockout allele of Ppp1r15a, we were unable to determine in which tissues loss of PPP1R15A is responsible for the food intake phenotype. We examined the expression of ISR targets, ATF4 and CHOP, in the hypothalamus by qRT-PCR but failed to detect significant differences (data not shown). Whilst this might argue against a central role for PPP1R15A, it is possible that our analysis of total hypothalamic mRNA missed differences between individual hypothalamic nuclei. Future studies using conditional deletion of Ppp1r15a or more focused analyses of hypothalamic nuclei will therefore be informative. Nevertheless, while this represents an important limitation of our study, the inactivation of PPP1R15A throughout the animal gives an indication of the effect PPP1R15A inhibition might have in a clinical setting. The complexity of the knockout phenotype, with better preservation of insulin sensitivity and yet higher peak glucose levels following glucose challenge, led us to question whether PPP1R15A antagonism would be beneficial or detrimental in models of diabetes. The direct role of PPP1R15A in insulin secretion in response to glucose remains to be addressed by more detailed assessments of in vivo glucose-stimulated insulin secretion. Future studies including frequently sampled intravenous glucose tolerance tests ${ }^{45}$, would help to elucidate the relative importance of PPP1R15A's effects on increased insulin sensitivity and reduced glucose clearance. Our finding that Ppp1r15a mutant mice depleted of most beta-cells with streptozotocin have improved glucose tolerance suggests that the beneficial effects on insulin sensitivity dominate in the setting of diabetes. This raises the exciting possibility that PPP1R15A inhibition may be a potential therapeutic strategy in obesity with insulin resistance or in advanced states of the natural evolution towards diet-induced diabetes.

We previously showed that loss of PPP1R15A ameliorated ER stress-induced nephrotoxicity ${ }^{12}$, which led us to speculate that inhibition of eIF $2 \alpha$ might protect beta-cell mass in the context of beta-cell exhaustion. Indeed, deletion of Chop, a transcription factor responsible for efficient induction of PPP1R15A, was shown to protect mice in variety of models of diabetes ${ }^{40,46}$. However, loss of Ppp1r15a has yet to be tested directly in an animal model of diabetes. Instead, efforts have focused on targeting PPP1R15A with small molecules, but these studies have been hampered by a lack of potent and selective molecules. Indeed, we recently demonstrated that the putative PPP1R15A inhibitors guanabenz and sephin1 do not affect PPP1R15A function, highlighting the urgent need for effective small molecules ${ }^{47}$. Salubrinal weakly inhibits dephosphorylation of eIF $2 \alpha$ at high concentrations ${ }^{47}$, but its dramatic effects to increase P-eIF $2 \alpha$ in cells may reflect additional effects perhaps on one or more of the eIF2 $\alpha$ kinases. In insulinoma cells, salubrinal was found to enhance the toxicity of palmitate ${ }^{48}$, but our data indicate that genetic loss of PPP1R15A ameliorates the lipotoxicity of palmitate. This supports a potential therapeutic role of PPP1R15A inhibition if more potent and selective small molecules were to be generated. 
In summary, we have observed that mice lacking the C-terminal PP1-binding domain of PPP1R15A are protected from obesity when fed a high-fat diet. This appears to reflect an increased satiety to diet, resulting in reduced weight gain and improved insulin sensitivity.

\section{Experimental Procedures}

Mice. The Guide for the care and use of laboratory animals, Eight edition (2011), was followed. The UK Home Office approved all animal protocols in this study (PPL 80/2491 \& PPL 80/2484). Generation of the Ppp1r15a $a^{\Delta C / \Delta C}$ mouse (originally named Ppp $1 r 15 a^{\text {tm1Dron }}$ ), has been described ${ }^{11}$. To minimise effects of genetic background, backcrossing was performed (Speed Congenics, Charles River, UK) until 97\% C57BL/6 purity. Mice were fed a standard chow diet (5.1\% fat, SAFE diets U8400G10R) or a semisynthetic high-fat diet (60\% fat, Research diets D12492i, Research Diets, New Brunswick, NJ). All mice used in this study were female. For treatment with streptozotocin, mice were intraperitoneally injected with $50 \mathrm{mg} / \mathrm{kg}$ streptozotocin (Sigma-S0130, Dorset, UK) freshly dissolved in sodium citrate $\mathrm{pH} 4.5$.

Metabolic profiling. We performed glucose tolerance tests after a 16-hour fast (5 p.m. to 8 a.m.) and insulin tolerance tests after a 4-hour fast ( 8 a.m. to 12 p.m.). Body composition was measured by time domain NMR (Bruker Optics). Daily food intake measurements were carried out on singly-housed mice in surgery bedding lined cages. Energy expenditure was measured by indirect-calorimetry in a Metatrace system (Creative Scientific, $\mathrm{UK}$ ) at $21^{\circ} \mathrm{C}$ attached to a custom-built oxygen and carbon dioxide monitoring system (Minimox system built by P. Murgatroyd). Carbon dioxide concentration in room air and air leaving each cage were measured every 11 minutes. Activity was assessed by beam breaks set $2.5 \mathrm{~cm}$ apart. Activity measurements were taken to be total beam breaks, measured every 8 minutes.

Glucose and insulin tolerance tests. Overnight fasted mice were injected intraperitoneally with $1 \mathrm{~g} / \mathrm{kg}$ glucose, calculated using the average bodyweight of the cohort, and blood glucose was measured for 2 hours. For the insulin tolerance tests, mice were fasted for 4 hours, basal glucose measured, and then mice were injected intraperitoneally with $0.75 \mathrm{U} / \mathrm{kg}$ of insulin, calculated using the average bodyweight of the $P p p 1 r 15 a^{\Delta C / \Delta C}$ cohort. Measurement of plasma insulin and free fatty acid was carried out using the Meso Scale Discovery (MSD) assays at the Core Biochemical Assay Laboratory (CBAL), Cambridge, UK.

Histology. Primary antibodies used were: guinea-pig anti-insulin (ab7842, 1:200; Abcam), rabbit anti-glucagon (SC13091, 1:200; SantaCruz, Santa Cruz, CA, USA). Sections were counterstained using Hoechst (1:1300, Invitrogen, UK). Stained sections were imaged using either the incubated Zeiss LSM510 META Confocal Microscope, Zeiss LSM710 META Confocal Microscope, Zeiss AxioImager Motorised Upright Microscope. Images were analysed using Zen software.

Cells and reagents. Wild type and Ppp1r15a $a^{\Delta C / \Delta C}$ MEFs were cultured as described ${ }^{12}$. Prior to experimentation, palmitate was first conjugated to BSA. Palmitic acid (Sigma-P0500, Dorset, UK) was prepared to a concentration of $100 \mathrm{mM}$ in $100 \%(\mathrm{v} / \mathrm{v})$ ethanol and heated at $60^{\circ} \mathrm{C}$. This was added to media supplemented with $5 \%$ $(\mathrm{w} / \mathrm{v})$ fatty-acid free, low endotoxin BSA (Sigma-A8806, Dorset, UK), to prepare a 10X stock and sonicated to ensure conjugation and solubilisation.

Immunoblots. Cell lysates were prepared and resolved as described ${ }^{12}$. Primary antibodies used were: rabbit anti-PPP1R15A (10449-1-AP, 1:1000; Proteintech, Manchester, UK), rabbit anti-phospho-eIF2 $\alpha$ (3597, 1:1000; Cell Signaling), mouse anti-actin (ab3280, 1:1000; Abcam), rabbit anti-ATF4 (C-20, 1:500; SantaCruz, Santa Cruz, CA, USA), mouse anti-total eIF2 $\alpha$ (AHO0802, 1:1000; Invitrogen, Thermo Fisher Scientific, Waltham, MA, USA); guinea pig anti-insulin (ab7842, 1:1000; Abcam), rabbit anti-GAPDH (14C10) (2118, 1:1000; Cell Signaling), rabbit anti-UCP1 (ab10983, 1:1000; Abcam), rabbit anti-CHOP (1:1000; kind gift from Professor David Ron, Cambridge, UK), rabbit anti-alpha/beta tubulin (2148, 1:1000; Cell Signalling, Hitchin, UK).

RNA extraction and real-time PCR. Total RNA was extracted from mammalian cells using the Qiagen RNeasy Mini Kit (Qiagen Ltd., UK), and from animal tissue using TRIzol reagent (15596026, ThermoFisher). cDNA was reverse transcribed with M-MLV Reverse Transcriptase (Invitrogen, UK), using an Oligo-(dt) 15 primer (Promega, UK). Real-time PCR was performed using an ABI7900-HT-Fast device (Applied Biosystems, Carlsbad, UK). Relative fold-changes of expression were determined using the relative standard curve method or, were stated in the legend, BestKeeper analysis ${ }^{49}$. Primer sequences used can be found in Supplementary Table S1.

Statistics. Data were analysed using unpaired Student's t-tests or analysis of variance (ANOVA) with Bonferroni post hoc correction (Prism, Graphpad, USA).

\section{References}

1. Chambers, J. E. \& Marciniak, S. J. Cellular mechanisms of endoplasmic reticulum stress signaling in health and disease. 2. Protein misfolding and ER stress. Am J Physiol Cell Physiol 307, C657-670, https://doi.org/10.1152/ajpcell.00183.2014 (2014).

2. Marciniak, S. J. \& Ron, D. Endoplasmic reticulum stress signaling in disease. Physiol Rev 86, 1133-1149, https://doi.org/10.1152/ physrev.00015.2006 (2006).

3. Ron, D. \& Walter, P. Signal integration in the endoplasmic reticulum unfolded protein response. Nat Rev Mol Cell Biol 8, 519-529, https://doi.org/10.1038/nrm2199 (2007).

4. Delepine, M. et al. EIF2AK3, encoding translation initiation factor 2-alpha kinase 3, is mutated in patients with Wolcott-Rallison syndrome. Nat Genet 25, 406-409 (2000).

5. Harding, H. et al. Diabetes Mellitus and excocrine pancreatic dysfunction in Perk $-/-$ mice reveals a role for translational control in survival of secretory cells. Mol Cell 7, 1153-1163 (2001). 
6. Harding, H. P., Zhang, Y. \& Ron, D. Protein translation and folding are coupled by an endoplasmic-reticulum-resident kinase. Nature 397, 271-274, https://doi.org/10.1038/16729 (1999).

7. Dalton, L. E., Healey, E., Irving, J. \& Marciniak, S. J. Phosphoproteins in stress-induced disease. Prog Mol Biol Transl Sci 106, 189-221, https://doi.org/10.1016/B978-0-12-396456-4.00003-1 (2012).

8. Lu, P. D., Harding, H. P. \& Ron, D. Translation re-initiation at alternative open reading frames regulates gene expression in an integrated stress response. J Cell Biol 167, 27-33 (2004).

9. Novoa, I., Zeng, H., Harding, H. P. \& Ron, D. Feedback inhibition of the unfolded protein response by GADD34-mediated dephosphorylation of eIF2alpha. J Cell Biol 153, 1011-1022 (2001).

10. Chambers, J. E. et al. Actin dynamics tune the integrated stress response by regulating eukaryotic initiation factor 2 alpha dephosphorylation. eLife 4 , https://doi.org/10.7554/eLife.04872 (2015).

11. Novoa, I. et al. Stress-induced gene expression requires programmed recovery from translational repression. $E M B O J \mathbf{2 2}, 1180-1187$, https://doi.org/10.1093/emboj/cdg112 (2003).

12. Marciniak, S. J. et al. CHOP induces death by promoting protein synthesis and oxidation in the stressed endoplasmic reticulum. Genes Dev 18, 3066-3077 (2004).

13. D’Antonio, M. et al. Resetting translational homeostasis restores myelination in Charcot-Marie-Tooth disease type 1B mice. J Exp Med 210, 821-838, https://doi.org/10.1084/jem.20122005 (2013).

14. Scheuner, D. et al. Control of mRNA translation preserves endoplasmic reticulum function in beta cells and maintains glucose homeostasis. Nat Med 11, 757-764 (2005).

15. Back, S. H. et al. Translation attenuation through eIF2alpha phosphorylation prevents oxidative stress and maintains the differentiated state in beta cells. Cell Metab 10, 13-26, https://doi.org/10.1016/j.cmet.2009.06.002 (2009).

16. Maurin, A. C. et al. Hypothalamic eIF2alpha signaling regulates food intake. Cell Rep 6, 438-444, https://doi.org/10.1016/j. celrep.2014.01.006 (2014).

17. Nishio, N. \& Isobe, K. GADD34-deficient mice develop obesity, nonalcoholic fatty liver disease, hepatic carcinoma and insulin resistance. Scientific reports 5, 13519, https://doi.org/10.1038/srep13519 (2015)

18. Cunha, D. A. et al. Initiation and execution of lipotoxic ER stress in pancreatic beta-cells. J Cell Sci 121, 2308-2318, https://doi. org $/ 10.1242 /$ jcs.026062 (2008).

19. Cnop, M. et al. Selective inhibition of eukaryotic translation initiation factor 2 alpha dephosphorylation potentiates fatty acidinduced endoplasmic reticulum stress and causes pancreatic beta-cell dysfunction and apoptosis. J Biol Chem 282, 3989-3997, https://doi.org/10.1074/jbc.M607627200 (2007).

20. Karaskov, E. et al. Chronic Palmitate but not Oleate Exposure Induces Endoplasmic Reticulum Stress Which May Contribute to INS-1 Pancreatic \{beta\}-cell Apoptosis. Endocrinology 147, 3398-3407 (2006).

21. Volmer, R., van der Ploeg, K. \& Ron, D. Membrane lipid saturation activates endoplasmic reticulum unfolded protein response transducers through their transmembrane domains. Proc Natl Acad Sci USA 110, 4628-4633, https://doi.org/10.1073/ pnas.1217611110 (2013).

22. Gwiazda, K. S., Yang, T. L., Lin, Y. \& Johnson, J. D. Effects of palmitate on ER and cytosolic Ca2+ homeostasis in beta-cells. Am J Physiol Endocrinol Metab 296, E690-701, https://doi.org/10.1152/ajpendo.90525.2008 (2009).

23. Nishio, N., Hasegawa, T., Tatsuno, I., Isaka, M. \& Isobe, K. I. Female GADD34 mice develop age-related inflammation and hepatocellular carcinoma. Geriatr Gerontol Int 17, 2593-2601, https://doi.org/10.1111/ggi.13080 (2017).

24. Oyadomari, S., Harding, H. P., Zhang, Y., Oyadomari, M. \& Ron, D. Dephosphorylation of translation initiation factor 2alpha enhances glucose tolerance and attenuates hepatosteatosis in mice. Cell metabolism 7, 520-532, https://doi.org/10.1016/j. cmet.2008.04.011 (2008).

25. Schroeder-Gloeckler, J. M. et al. CCAAT/enhancer-binding protein beta deletion reduces adiposity, hepatic steatosis, and diabetes in Lepr(db/db) mice. J Biol Chem 282, 15717-15729, https://doi.org/10.1074/jbc.M701329200 (2007).

26. Millward, C. A. et al. Mice with a deletion in the gene for CCAAT/enhancer-binding protein beta are protected against diet-induced obesity. Diabetes 56, 161-167, https://doi.org/10.2337/db06-0310 (2007).

27. Prentki, M. \& Nolan, C. J. Islet beta cell failure in type 2 diabetes. J Clin Invest 116, 1802-1812, https://doi.org/10.1172/JCI29103 (2006).

28. Liu, X. et al. Paradoxical resistance to diet-induced obesity in UCP1-deficient mice. J Clin Invest 111, 399-407, https://doi. org/10.1172/JCI15737 (2003)

29. Eckel, L. A. The ovarian hormone estradiol plays a crucial role in the control of food intake in females. Physiol Behav 104, 517-524, https://doi.org/10.1016/j.physbeh.2011.04.014 (2011).

30. Gonzalez-Garcia, I. et al. Estradiol Regulates Energy Balance by Ameliorating Hypothalamic Ceramide-Induced ER Stress. Cell Rep 25, 413-423 e415, https://doi.org/10.1016/j.celrep.2018.09.038 (2018).

31. Chen, R. et al. G-actin provides substrate-specificity to eukaryotic initiation factor 2alpha holophosphatases. eLife 4, https://doi. org/10.7554/eLife.04871 (2015).

32. Harding, H. P. et al. Ppp1r15 gene knockout reveals an essential role for translation initiation factor 2 alpha (eIF2alpha) dephosphorylation in mammalian development. Proc Natl Acad Sci USA 106, 1832-1837, https://doi.org/10.1073/pnas.0809632106 (2009).

33. Abdulkarim, B. et al. A Missense Mutation in PPP1R15B Causes a Syndrome Including Diabetes, Short Stature, and Microcephaly. Diabetes 64, 3951-3962, https://doi.org/10.2337/db15-0477 (2015)

34. Ozcan, U. et al. Endoplasmic reticulum stress links obesity, insulin action, and type 2 diabetes. Science 306, 457-461 (2004).

35. Ozcan, U. et al. Chemical chaperones reduce ER stress and restore glucose homeostasis in a mouse model of type 2 diabetes. Science 313, 1137-1140, https://doi.org/10.1126/science.1128294 (2006).

36. Laybutt, D. R. et al. Endoplasmic reticulum stress contributes to beta cell apoptosis in type 2 diabetes. Diabetologia 50, 752-763, https://doi.org/10.1007/s00125-006-0590-z (2007).

37. Scheuner, D. et al. Translational control is required for the unfolded protein response and in-vivo glucose homeostasis. Mol Cell 7, $1165-1176(2001)$.

38. Bi, M. et al. ER stress-regulated translation increases tolerance to extreme hypoxia and promotes tumor growth. EMBO J 24, 3470-3481, https://doi.org/10.1038/sj.emboj.7600777 (2005).

39. Atkins, C. et al. Characterization of a novel PERK kinase inhibitor with antitumor and antiangiogenic activity. Cancer Res $\mathbf{7 3}$, 1993-2002, https://doi.org/10.1158/0008-5472.CAN-12-3109 (2013).

40. Oyadomari, S. et al. Targeted disruption of the Chop gene delays endoplasmic reticulum stress-mediated diabetes. J Clin Invest 109, 525-532 (2002)

41. Sowers, C. R. et al. The protein kinase PERK/EIF2AK3 regulates proinsulin processing not via protein synthesis but by controlling endoplasmic reticulum chaperones. J Biol Chem 293, 5134-5149, https://doi.org/10.1074/jbc.M117.813790 (2018)

42. Birkenfeld, A. L. et al. Influence of the hepatic eukaryotic initiation factor 2alpha (eIF2alpha) endoplasmic reticulum (ER) stress response pathway on insulin-mediated ER stress and hepatic and peripheral glucose metabolism. J Biol Chem 286, 36163-36170, https://doi.org/10.1074/jbc.M111.228817 (2011).

43. Maurin, A. C. et al. The GCN2 kinase biases feeding behavior to maintain amino acid homeostasis in omnivores. Cell Metab 1, 273-277, https://doi.org/10.1016/j.cmet.2005.03.004 (2005). 
44. Horwath, J. A. et al. Obesity-induced hepatic steatosis is mediated by endoplasmic reticulum stress in the subfornical organ of the brain. JCI Insight 2, https://doi.org/10.1172/jci.insight.90170 (2017).

45. Alonso, L. C. et al. Simultaneous measurement of insulin sensitivity, insulin secretion, and the disposition index in conscious unhandled mice. Obesity (Silver Spring) 20, 1403-1412, https://doi.org/10.1038/oby.2012.36 (2012).

46. Song, B., Scheuner, D., Ron, D., Pennathur, S. \& Kaufman, R. J. Chop deletion reduces oxidative stress, improves beta cell function, and promotes cell survival in multiple mouse models of diabetes. JClin Invest 118, 3378-3389, https://doi.org/10.1172/JCI34587 (2008).

47. Crespillo-Casado, A., Chambers, J. E., Fischer, P. M., Marciniak, S. J. \& Ron, D. PPP1R15A-mediated dephosphorylation of eIF2alpha is unaffected by Sephin1 or Guanabenz. eLife 6, e26109, https://doi.org/10.7554/eLife.26109.001 (2017).

48. Fransson, L., Sjoholm, A. \& Ortsater, H. Inhibition of palmitate-induced GADD34 expression augments apoptosis in mouse insulinoma cells (MIN6). Cell Biochem Funct 32, 445-452, https://doi.org/10.1002/cbf.3036 (2014).

49. Pfaffl, M. W., Tichopad, A., Prgomet, C. \& Neuvians, T. P. Determination of stable housekeeping genes, differentially regulated target genes and sample integrity: BestKeeper-Excel-based tool using pair-wise correlations. Biotechnol Lett 26, 509-515 (2004).

\section{Acknowledgements}

We would like to thank Sarah Grocott and Dan Hart for technical support. The work was also supported by Diabetes UK and the MRC [G1002610]. VP held an Arthur and Sadie Pethybridge PhD Studentship from Diabetes UK. The CIMR microscopy core facility is supported by a Wellcome Trust Strategic Award [100140] and a Wellcome Trust equipment grant [093026].

\section{Author Contributions}

V.P. designed and performed the majority of experiments presented. S.J.M. conceived the project as a whole. G.B. designed and helped to perform and anlyse experiments addressing the metabolic phenotype of the Ppp1r15a mutant mice. J.E.C., S.C., A.J.T.E., C.G. and L.E.D. contributed to experimental design and performance throughout. F.M.G., A.V.P. and S.J.M. oversaw the project. All authors contributed to writing the manuscript.

\section{Additional Information}

Supplementary information accompanies this paper at https://doi.org/10.1038/s41598-019-39562-y.

Competing Interests: The authors declare no competing interests.

Publisher's note: Springer Nature remains neutral with regard to jurisdictional claims in published maps and institutional affiliations.

(c) (i) Open Access This article is licensed under a Creative Commons Attribution 4.0 International License, which permits use, sharing, adaptation, distribution and reproduction in any medium or format, as long as you give appropriate credit to the original author(s) and the source, provide a link to the Creative Commons license, and indicate if changes were made. The images or other third party material in this article are included in the article's Creative Commons license, unless indicated otherwise in a credit line to the material. If material is not included in the article's Creative Commons license and your intended use is not permitted by statutory regulation or exceeds the permitted use, you will need to obtain permission directly from the copyright holder. To view a copy of this license, visit http://creativecommons.org/licenses/by/4.0/.

(c) The Author(s) 2019 